\title{
Discovery of A Binary System in IRAM 04191+1522
}

\author{
Xuepeng Chen ${ }^{1}$, Héctor G. Arce ${ }^{1}$, Michael M. Dunham ${ }^{1}$, and Qizhou Zhang ${ }^{2}$ \\ ${ }^{1}$ Department of Astronomy, Yale University, Box 208101, New Haven, CT 06520-8101, \\ USA; xuepeng.chen@yale.edu \\ ${ }^{2}$ Harvard-Smithsonian Center for Astrophysics, 60 Garden Street, Cambridge, MA 02138, \\ USA
}

\begin{abstract}
We present high angular resolution observations of the Class 0 protostar IRAM 04191+1522, using the Submillimeter Array (SMA). The SMA $1.3 \mathrm{~mm}$ continuum images reveal within IRAM 04191+1522 two distinct sources with an angular separation of $7.8 \pm 0.2^{\prime \prime}$. The two continuum sources are located in the southeast-northwest direction, with total gas masses of $\sim 0.011 M_{\odot}$ and $\sim 0.005 M_{\odot}$, respectively. The southeastern source, associated with an infrared source seen in the Spitzer images, is the well-known Class 0 protostar with a bolometric luminosity of $\sim 0.08 L_{\odot}$. The newly-discovered northwestern continuum source is not visible in the Spitzer images at wavelengths from 3.6 to $70 \mu \mathrm{m}$, and has an extremely low bolometric luminosity $\left(<0.03 L_{\odot}\right)$. Complementary IRAM $\mathrm{N}_{2} \mathrm{H}^{+}(1-0)$ data that probe the dense gas in the common envelope suggest that the two sources were formed through the rotational fragmentation of an elongated dense core. Furthermore, comparisons between IRAM 04191+1522 and other protostars suggest that most cores with binary systems formed therein have ratios of rotational energy to gravitational energy $\beta_{\text {rot }}>1 \%$. This is consistent with theoretical simulations and indicates that the level of rotational energy in a dense core plays an important role in the fragmentation process.
\end{abstract}

Subject headings: binaries: general - ISM: clouds - ISM: individual (IRAM 04191+1522) - stars: formation

\section{INTRODUCTION}

The origin of binary stars is one of the major puzzles in our understanding of star formation. Binary and higher-order multiple systems have been frequently observed in all stages of stellar evolution, but at present we do not understand well how this occurs (see, e.g., 
reviews by Tohline 2002; Goodwin et al. 2007). The formation of binary systems begins in the earliest phase of the star formation process, when the young protostar, generally referred to as Class 0 protostar (André et al. 2000), is still deeply embedded within a dense core of gas and dust. In order to achieve a comprehensive understanding of binary star formation, high angular resolution observations of the gas and optically thin dust emission at (sub-) millimeter wavelengths are therefore required to probe the system kinematics and individual core masses. However, these observations were long limited by the low angular resolution of single-dish millimeter telescopes, and have only become possible with the recent availability of large (sub-) millimeter interferometers.

In the past decade, a handful of Class 0 protostellar binary (protobinary) systems have been found using different interferometers (e.g., Looney et al. 2000; Launhardt 2004), and there are increasing interferometric studies of binarity in the protostellar phase (e.g., Volgenau et al. 2006; Chen et al. 2007; Maury et al. 2010). Nevertheless, due to the large effort and observing time required, the number of known and well-studied protobinary systems is still small. Furthermore, high angular resolution kinematics data, which could be used to study the physical process leading to the formation of binary stars, are also lacking. Therefore, it is critical to observe, at high angular resolution, more protostellar cores in nearby molecular clouds to search for protobinary candidates and study in detail their kinematic properties, and then compare the observational results with different theoretical models.

In this Letter, we present interferometric observations of IRAM 04191+1522 (hereafter IRAM 04191), using the Submillimeter Array 1 (SMA; Ho et al. 2004). IRAM 04191 is a Class 0 protostar discovered in the southern part of the Taurus molecular cloud (André et al. 1999). From its low bolometric luminosity $\left(L_{\mathrm{bol}} \sim 0.15 L_{\odot}\right)$ and temperature $\left(T_{\mathrm{bol}} \sim 18 \mathrm{~K}\right)$, André et al. (1999) estimated an age of $\sim 1-3 \times 10^{4} \mathrm{yr}$, suggesting IRAM 04191 as one of the youngest protostars in Taurus. IRAM 04191 drives a large scale $(\sim 0.1 \mathrm{pc})$, bipolar molecular outflow, which extends in the northeast-southwest direction (André et al. 1999; Lee et al. 2005). Various molecular line observations have shown subsonic infall, fast rotation, depletion, and deuteration in IRAM 04191 (see, e.g., Belloche et al. 2002; Takakuwa et al. 2003; Belloche \& André 2004; Lee et al. 2005). This source is also detected in the Spitzer Space Telescope (Spitzer) images at wavelengths between 3.6 and $70 \mu \mathrm{m}$, and is classified as a very low luminosity object (VeLLO), due to its low internal luminosity $2\left(L_{\text {int }} \sim 0.08 L_{\odot}\right.$;

\footnotetext{
${ }^{1}$ The Submillimeter Array is a joint project between the Smithsonian Astrophysical Observatory and the Academia Sinica Institute of Astronomy and Astrophysics and is funded by the Smithsonian Institution and the Academia Sinica.

${ }^{2}$ The internal luminosity is the luminosity of the central source, which excludes the luminosity arising from external heating.
} 
Dunham et al. 2006, 2008). Based on the SMA observations, we report in this Letter that IRAM 04191, regarded as an excellent example of isolated single star formation in the past decade, is actually a binary system, which may in turn represent one of the best examples of a protobinary system formed though rotational fragmentation.

\section{OBSERVATIONS AND DATA REDUCTION}

The SMA $230 \mathrm{GHz}$ observations of IRAM 04191 were carried out in 2007 November $(\sim 5$ hours integration time) with the compact configuration (PI: J. Karr), and in 2011 March $(\sim 1$ hour integration time) with the subcompact configuration (PI: X. Chen). Seven antennas were used in both configurations, providing baselines ranging from 9-53 k $\lambda$ (compact) and 6$51 \mathrm{k} \lambda$ (subcompact), respectively. In the 2007 observations, the digital correlator was set up to cover the frequencies from 219.5 to $221.4 \mathrm{GHz}$ and from 229.5 to $231.4 \mathrm{GHz}$ in the lower and upper sidebands (LSB and USB), respectively. The $1.3 \mathrm{~mm}$ continuum emission was recorded with a total bandwidth of $\sim 3.12 \mathrm{GHz}$, combining the line-free portions of the two sidebands. In the 2011 observations, the SMA bandwidth was upgraded to $4 \mathrm{GHz}$, and the correlator was set up to cover the frequencies from 216.8 to $220.8 \mathrm{GHz}$ (LSB) and from 228.8 to $232.8 \mathrm{GHz}$ (USB), respectively, resulting in a total continuum bandwidth of $\sim 7.46 \mathrm{GHz}$. The SMA primary beam is about $55^{\prime \prime}$ at $230 \mathrm{GHz}$.

The visibility data were calibrated with the MIR package (Qi 2005). In the 2007 observations, the quasar 3c454.3 was used for bandpass calibration, and quasars 3c120 and $0530+135$ for gain calibration. In the 2011 observations, the quasar $0854+201$ was used for bandpass calibration, and quasars 3c84 and 0359+509 for gain calibration. Uranus was used for absolute flux calibration in the 2007 observations, while 0854+201 was used in the 2011 observations. We estimate a flux accuracy of $\sim 20 \%$ for the data, by comparing the final quasar fluxes with the SMA calibration database. The calibrated visibility data were further imaged using the Miriad toolbox (Sault et al. 1995).

\section{RESULTS}

Figure 1 shows the SMA $1.3 \mathrm{~mm}$ dust continuum images (top panel) and the Spitzer infrared images (bottom panel) of IRAM 04191. The SMA $1.3 \mathrm{~mm}$ dust continuum observations reveal within IRAM 04191 two distinct sources, which are separated by $7.8 \pm 0.2^{\prime \prime}$ in the northwest-southeast direction (see Figures 1a-c). These two sources were detected with the SMA compact and subcompact configurations independently, thus yielding a solid de- 
tection. The SMA compact configuration observations detected relatively compact emission clearly arising from two distinct sources (see Figure 1a), while the subcompact configuration observations detected relatively extended emission with two local maxima (see Figure 1b), likely tracing an inner common envelope. As shown in the Spitzer images (see Figures 1df), the southeastern continuum source is associated with an infrared source, and is therefore referred to as IRAM 04191 IRS in this work (see also Dunham et al. 2006). In contrast, the northwestern continuum source has no compact infrared emission at the Spitzer bands from 3.6 to $70 \mu \mathrm{m}$, and is referred to as IRAM $04191 \mathrm{MMS} 3$. The comparisons with previous high angular resolution observations (e.g., Belloche et al. 2002; Lee et al. 2005) suggest that source IRS is the well-known Class 0 protostar, which drives a large scale bipolar outflow in the northeast-southwest direction.

The continuum fluxes of the two sources were derived from Gaussian fits to the restored images (using the combined compact and subcompact data) with the Miriad command imfit (see Table 1). The fluxes of sources IRAM 04191 IRS and MMS are $\sim 9.3 \mathrm{mJy}$ and $\sim 4.7 \mathrm{mJy}$, respectively. For comparison, the continuum flux detected in the IRAM-30m $1.3 \mathrm{~mm}$ map is $\sim 650$ mJy (aperture 60"; André et al. 1999), indicating that more than 95\% of the flux from the large-scale envelope around the two sources was resolved out by the SMA observations. Furthermore, it must be noted that in the IRAM Plateau de Bure Interferometer (PdBI) $1.3 \mathrm{~mm}$ continuum observations, only a single object was detected in IRAM 04191, which is spatially coincident with source IRAM 04191 IRS (see Maury et al. 2010). It is likely that the PdBI observations missed the relatively faint source MMS (with about half of the IRS flux), due to the low flux ratio sensitivity in the IRAM 04191 observations (only about 0.6-0.9; Maury et al. 2010).

Assuming that the $1.3 \mathrm{~mm}$ dust continuum emission is optically thin, the total gas mass $\left(M_{\text {gas }}\right)$ of the two sources is derived from their fluxes using the same method as described in Launhardt \& Henning (1997). In the calculations, we adopt a dust opacity of $\kappa_{\mathrm{d}}=$ $0.5 \mathrm{~cm}^{2} \mathrm{~g}^{-1}$ (Ossenkopf \& Henning 1994), a typical value for dense and cold molecular cloud cores. A dust temperature of $\sim 20 \mathrm{~K}$ was adopted for IRAM $04191 \mathrm{MMS}$ and IRS, which is derived from fitting the SEDs (see below). The relative uncertainties of the derived masses due to the calibration errors of the fluxes are $\pm 20 \%$. The total gas masses of IRAM 04191 IRS and MMS derived from the SMA maps are $\sim 0.011 M_{\odot}$ and $\sim 0.005 M_{\odot}$, respectively.

\footnotetext{
${ }^{3}$ The Spitzer spatial resolution is $\sim 18^{\prime \prime}$ at $70 \mu \mathrm{m}$, which fails to resolve sources IRS and MMS. However, due to the facts that (1) the $70 \mu \mathrm{m}$ emission peaks at source IRS, without any shift toward source MMS, and (2) the emission is roughly circularly symmetric (see Figure 1f), we consider that all the $70 \mu \mathrm{m}$ emission comes only from source IRS.
} 


\section{DISCUSSION}

\subsection{The Spectral Energy Distributions}

In order to understand the properties of sources IRAM 04191 IRS and MMS, it is essential to construct their spectral energy distributions (SEDs), and then to derive their luminosities and temperatures. However, previous single-dish (sub-) millimeter continuum observations lack the resolution to resolve the two sources. Therefore, we simply assume a flux ratio of $2: 1$ at these wavelengths (from $160 \mu \mathrm{m}$ to $1.3 \mathrm{~mm}$ ), which is inferred from the SMA flux ratio of the two sources. Furthermore, due to the fact that no compact infrared emission was detected from MMS in the Spitzer 3.6 to $70 \mu \mathrm{m}$ bands but infrared emission was detected at the position of IRS, we assume that all the infrared flux at wavelengths $\leq 90 \mu \mathrm{m}$ comes from source IRS. Given these assumptions, we show in Figure 2 the SEDs of sources IRS and MMS (see Dunham et al. 2008 for individual flux values). To derive luminosities and temperatures, we first interpolated and then integrated the SEDs, assuming spherical symmetry. Interpolation between the flux densities was done by a $\chi^{2}$ single-temperature grey-body fit to all points at $\lambda>90 \mu \mathrm{m}$, using the method described in Chen et al. (2008). A simple logarithmic interpolation was performed between all points at $\lambda \leq 90 \mu \mathrm{m}$ (including $3 \sigma$ upper limits for source MMS). From the SED fitting, we estimate $L_{\mathrm{bol}} \sim 0.08 L_{\odot}$ and $T_{\mathrm{bol}} \sim 25 \mathrm{~K}$ for source IRS, and $L_{\mathrm{bol}}<0.03 L_{\odot}$ and $T_{\mathrm{bol}}<20 \mathrm{~K}$ for source MMS. The dust temperature estimated for the two sources is $20 \pm 3 \mathrm{~K}$. Further observations, such as high angular resolution and high sensitivity continuum observations at wavelengths from far-infrared to (sub-) millimeter, are needed to constrain the SEDs of IRAM 04191 IRS and MMS, in order to derive more precisely their luminosities and temperatures.

\subsection{A Case of Rotational Fragmentation}

It has been suggested that the fragmentation of rotationally flattened cloud cores with initially flat density profiles, immediately after a phase of free-fall collapse, is one of the most efficient mechanisms for binary star formation (see reviews by Tohline 2002 and Goodwin et al. 2007). Numerical simulations find that rotating cloud cores fragment if $\alpha_{\text {therm }} \beta_{\text {rot }}$ $\leq 0.12-0.15$ (e.g., Tohline 1981), where $\alpha_{\text {therm }}=E_{\text {therm }} / E_{\text {grav }}$ is the initial thermal virial ratio ( $E_{\text {therm }}$ is the thermal kinetic and $E_{\text {grav }}$ is the gravitational potential energy) and $\beta_{\text {rot }}$ $=E_{\text {rot }} / E_{\text {grav }}$ is the initial rotational virial ratio $\left(E_{\text {rot }}\right.$ is the rotational kinetic energy). In particular, the simulations find that the level of rotation energy, i.e., $\beta_{\text {rot }}$, plays an important role in this fragmentation process. For example, Boss (1999) found that rotating cloud cores fragment only when $\beta_{\text {rot }}>0.01$ (see also Machida et al. 2005). 
Figure 3 shows the velocity field of IRAM 04191, using the combined IRAM-30m and PdBI $\mathrm{N}_{2} \mathrm{H}^{+}$(1-0) data from Belloche \& André (2004) and derived with the same method used by Chen et al. (2007). The mean velocity map shows a clear continuous velocity gradient across the dense core, increasing from northwest to southeast, i.e., roughly along the line connecting sources MMS and IRS (see Figure 3a). As discussed in Chen et al. (2007), systematic velocity gradients found in protostellar cores/envelopes are usually dominated by either rotation or outflow. As seen in Figure $3 \mathrm{a}$, the large angle $\left(>70^{\circ}\right)$ between the gradient and the axis of the IRS outflow suggests that the observed velocity gradient in $\mathrm{N}_{2} \mathrm{H}^{+}$is due to core rotation rather than outflow. Figure $3 \mathrm{~b}$ shows the spatial distribution of the $\mathrm{N}_{2} \mathrm{H}^{+}$ line widths in the map. The line widths are roughly constant within the core, and relatively large line widths are mainly seen in the central region of the core (roughly along the outflow axis), which might be caused by the outflow (see Chen et al. 2007 for more discussions).

From the velocity field derived from the $\mathrm{N}_{2} \mathrm{H}^{+}(1-0)$ observations, we can estimate the ratios of rotational and thermal energy to gravitational potential energy, using equations obtained from Chen et al. (2007; 2008):

$$
\begin{gathered}
\beta_{\text {rot }}=0.263 \times\left(\frac{R}{\mathrm{pc}}\right)^{2} \times\left(\frac{g}{\mathrm{~km} \mathrm{~s}^{-1} \mathrm{pc}^{-1}}\right)^{2} \times\left(\frac{\mathrm{km} \mathrm{s}^{-1}}{\Delta v_{\mathrm{obs}}}\right)^{2}, \\
\alpha_{\text {therm }}=0.01 \times\left(\frac{T}{\mathrm{~K}}\right) \times\left(\frac{\mathrm{km} \mathrm{s}^{-1}}{\Delta v_{\mathrm{obs}}}\right)^{2},
\end{gathered}
$$

where $R$ is the core radius (the FWHM radius $\sim 20^{\prime \prime}$ or $2800 \mathrm{AU}$ ), $g$ is the velocity gradient $\left(\sim 17 \mathrm{~km} \mathrm{~s}^{-1} \mathrm{pc}^{-1}\right), T$ is the kinetic gas temperature (6-10 K; see Belloche et al. 2002), and $\Delta v_{\text {obs }}$ is the observed mean line width $\left(0.50-0.55 \mathrm{~km} \mathrm{~s}^{-1}\right.$; see Figure $\left.3 \mathrm{~b}\right)$. The estimated $\beta_{\text {rot }}$ and $\alpha_{\text {therm }}$ values for IRAM 04191 are $0.06 \pm 0.02$ and $0.3 \pm 0.1$, respectively. Correcting for inclination $\left(i=50^{\circ}\right.$; see Belloche et al. 2002), the estimated $\beta_{\text {rot }}$ is then about 0.17 , consistent with the value of $\sim 0.1-0.2$ derived by Belloche et al. (2002). It is clear from these values that IRAM 04191 meets the classical fragmentation criterion of $\alpha_{\text {therm }} \beta_{\text {rot }} \leq$ 0.12 , and the estimated $\beta_{\text {rot }}$ value is far beyond the critical value suggested by numerical simulations. We thus suggest that the two sources in IRAM04191 were formed through the rotational fragmentation of an elongated dense core. Furthermore, because of its clear rotational picture, close distance, and youth, as well as its other well-characterized properties derived from previous observations, IRAM 04191 represents so far one of the best examples to observationally study the rotational fragmentation of dense cores.

Interestingly, Tohline (1981) pointed out that the semi-major axis of a binary formed through rotational fragmentation is

$$
a_{\text {bin }}=\left(\frac{4}{\pi}\right) \beta_{\text {rot }} R_{\text {core }}
$$


For IRAM 04191, its core radius derived from the IRAM-30m $1.3 \mathrm{~mm}$ observations is about $4200 \mathrm{AU}$ or $30^{\prime \prime}$ (see André et al. 1999). Adopting $\beta_{\text {rot }}=0.17$, the predicted binary separation is $\sim 6.5^{\prime \prime}$, close to the value measured in the SMA $1.3 \mathrm{~mm}$ continuum images $\left(\sim 7.8^{\prime \prime}\right)$.

\subsection{The Role of Rotation in Binary Star Formation}

At this stage, it is instructive to compare IRAM 04191 to other protostars with high angular resolution kinematics data, and then to observationally investigate the role of rotation in binary star formation. Based on our previous observations (Chen et al. 2007; 2008; 2009), we have collected high angular resolution $\mathrm{N}_{2} \mathrm{H}^{+}(1-0)$ data for twelve Class 0 protostars. Among those protostars, two protobinary systems, CB 230 (Chen et al. 2007) and SVS 13B (Chen et al. 2009), show a similar rotational picture as seen in IRAM 04191, and may represent another two cases of rotational fragmentation. In addition, $\mathrm{VLA} \mathrm{NH}_{3}$ observations suggest core/envelope rotation in the protostars HH 212-MMS (Wiseman et al. 2001) and HH 211-mm (Tanner \& Arce 2011), both of which were also resolved into binary systems in high angular resolution observations (Codella et al. 2007; Lee et al. 2009). More recently, Tobin et al. (2011) present high angular resolution $\mathrm{N}_{2} \mathrm{H}^{+}$and $\mathrm{NH}_{3}$ data for another eleven protostars. With these kinematic data, we estimate the $\beta_{\text {rot }}$ values for the individual sources (using the same method given above), and show in Figure 4 the distribution of $\beta_{\text {rot }}$ versus core FWHM radius for those sources (more analyses based on these kinematics data will be presented in a forthcoming paper).

As seen in Figure 4, we find that most cores with binary systems formed therein have $\beta_{\text {rot }}>1 \%$. In contrast, those cores with $\beta_{\text {rot }}<1 \%$, such as the well-studied protostar L1157, remain as single protostars in the observations. This is consistent with the theoretical simulations (see above) and indicates that the level of rotational energy in a dense core plays an important role in the fragmentation process. Nevertheless, a few protostars with $\beta_{\text {rot }}>$ $1 \%$ also remain as single (see Figure 4). We speculate that these protostars are unresolved binary/multiple systems due to the limitation of angular resolution or sensitivity. Indeed, for example, our SMA $\mathrm{HCO}^{+}$and $\mathrm{CO}$ observations suggest that one such protostar, L1521F, is likely a multiple system with a quadrupolar outflow (X. Chen et al. in preparation). However, it must be noted that (1) the size of the sample in Figure 4 is still small and no statistically significant conclusion can be drawn yet; and (2) more high angular resolution observations are needed to study a larger sample thoroughly and to investigate if other core properties, e.g., turbulence, density structure, and magnetic field, also have effects on the fragmentation process. 


\section{SUMMARY}

We report the discovery of a binary system with an angular separation of $7.8 \pm 0.2^{\prime \prime}$ in the well-known Class 0 protostar IRAM 04191, using the SMA observations. Complementary IRAM $\mathrm{N}_{2} \mathrm{H}^{+}(1-0)$ data suggest that the binary system is formed through the rotational fragmentation of an elongated dense core. Furthermore, comparisons between IRAM 04191 and other protostellar cores suggest that most cores with a binary system formed therein have ratios of rotational energy to gravitational energy $\beta_{\text {rot }}>1 \%$, which is consistent with theoretical simulations and indicates that the level of rotational energy in a dense core plays an important role in the fragmentation process.

We thank the anonymous referee for many insightful comments and suggestions. We thank A. Belloche and P. André for providing their $\mathrm{N}_{2} \mathrm{H}^{+}(1-0)$ data of IRAM 04191+1522. This material is based on work supported by NSF grant AST-0845619 to HGA. 


\section{REFERENCES}

André, P., Motte, F., \& Bacmann, A. 1999, ApJ, 513, L57

André, P., Ward-Thompson, D., \& Barsony, M. 2000, in Protostars and Planets IV, ed. V. Mannings, A. P. Boss, \& S. S. Russell (Tucson: Univ. Arizona Press), 59

Belloche, A., \& André, P. 2004, A\&A, 419, L35

Belloche, A., André, P., Despois, D., \& Blinder, S. 2002, A\&A, 393, 927

Belloche, A., Hennebelle, P., \& André, P. 2006, A\&A, 453, 145

Boss, A. P. 1999, ApJ, 520, 744

Chen, X., Launhardt, R., \& Bourke, T. L., Henning, Th., \& Barnes, P. J. 2008, ApJ, 683, 862

Chen, X., Launhardt, R., \& Henning, Th. 2007, ApJ, 669, 1058

Chen, X., Launhardt, R., \& Henning, Th. 2009, ApJ, 691, 1729

Codella, C., Cabrit, S., Gueth, F., Cesaroni, R., \& Bacciotti, F., et al. 2007, A\&A, 462, L53

Dunham, M. M., Crapsi, A., Evans II, N. J., Bourke, T. L., \& Huard, T. L., et al. 2008, ApJS, 179, 249

Dunham, M. M., Evans II, N. J., Bourke, T. L., Dullemond, C. P., \& Young, C. H., et al. 2006, ApJ, 651, 945

Goodwin, S., Kroupa, P., Goodman, A., \& Burkert A. 2007, in Protostars and Planets V, ed. B. Reipurth, D. Jewitt, \& K. Keil (Tucson: Univ. Arizona Press), 133

Ho, P. T. P., Moran, J. M., \& Lo, K. Y. 2004, ApJ, 616, L1

Launhardt, R. 2004, in IAU Symp. 221, Star Formation at High Angular Resolution, ed. M. G. Burton, R. Jayawardhana, \& T. L. Bourke (San Francisco: ASP), 213

Launhardt, R., \& Henning, Th. 1997, A\&A, 326, 329

Lee, C.-F., Hirano, N., Palau, A., Ho, P. T. P., \& Bourke, T. L., et al. 2009, ApJ, 699, 1584

Lee, C.-F., Ho, P. T. P., \& White, S. M. 2005, ApJ, 619, 948

Looney, L. W., Mundy, L. G., \& Welch, W. J. 2000, ApJ, 529, 477 
Machida, M. N., Matsumoto, T., Hanawa, T., \& Tomisaka, K. 2005, MNRAS, 362, 382

Maury, A. J., André, Ph., Hennebelle, P., Motte, F., \& Stamatellos, D., et al. 2010, A\&A, 512, 40

Ossenkopf, V., \& Henning, Th. 1994, A\&A, 291, 943

Qi, C. 2005, MIR Cookbook (Cambridge: Harvard), http://cfa-www.harvard.edu/ cqi/mircook.html

Sault, R. J., Teuben, P. J., \& Wright, M. C. H. 1995, in ASP Conf. Ser. 77, Astronomical Data Analysis Software and Systems IV, ed. R. A. Shaw, H. E. Payne, \& J. J. E. Hayes (San Francisco: ASP), 443

Takakuwa, S., Ohashi, N., \& Hirano, N. 2003, ApJ, 590, 932

Tanner, J. D., \& Arce, H. G. 2011, ApJ, 726, 40

Tobin, J. J., Hartmann, L., Chiang, H.-F., Looney, L. W., \& Bergin, E. A., et al. 2011, ApJ, 740,45

Tohline, J. E. 1981, ApJ, 248, 717

Tohline, J. E. 2002, ARA\&A, 40, 349

Volgenau, N. H., Mundy, L. G., Looney, L. W., \& Welch, W. J. 2006, ApJ, 651, 301

Wiseman, J., Wootten, A., Zinnecker, H., \& McCaughrean, M. 2001, ApJ, 550, L87 
Table 1. SMA $1.3 \mathrm{~mm}$ dust continuum results of IRAM 04191

\begin{tabular}{cccccc}
\hline \hline $\begin{array}{c}\text { Source } \\
\text { Name }\end{array}$ & $\begin{array}{c}\text { R.A. \& Dec. }(\mathrm{J} 2000) \\
{\left[\mathrm{h}: \mathrm{m}: \mathrm{s},{ }^{\circ}:^{\prime}:{ }^{\prime \prime}\right]}\end{array}$ & $\begin{array}{c}\text { Flux }^{a} \\
{[\mathrm{mJy}]}\end{array}$ & FWHM Sizes $^{a}$ & \multirow{2}{*}{$\begin{array}{c}M_{\text {gas }}{ }^{b} \\
{\left[M_{\odot}\right]}\end{array}$} \\
\hline IRAM 04191 IRS & $04: 21: 56.90,15: 29: 46.4$ & $9.3 \pm 2.0$ & $3.6^{\prime \prime} \times 3.5^{\prime \prime}$ & & $0.011 \pm 0.002$ \\
IRAM 04191 MMS & $04: 21: 56.37,15: 29: 48.9$ & $4.7 \pm 1.0$ & $2.5^{\prime \prime} \times 1.6^{\prime \prime}$ & $0.005 \pm 0.001$ \\
\hline
\end{tabular}

${ }^{\text {a}}$ Flux and FWHM sizes derived from the Gaussian fitting.

${ }^{\mathrm{b}}$ Total gas mass; See text for dust temperature and opacity used. 

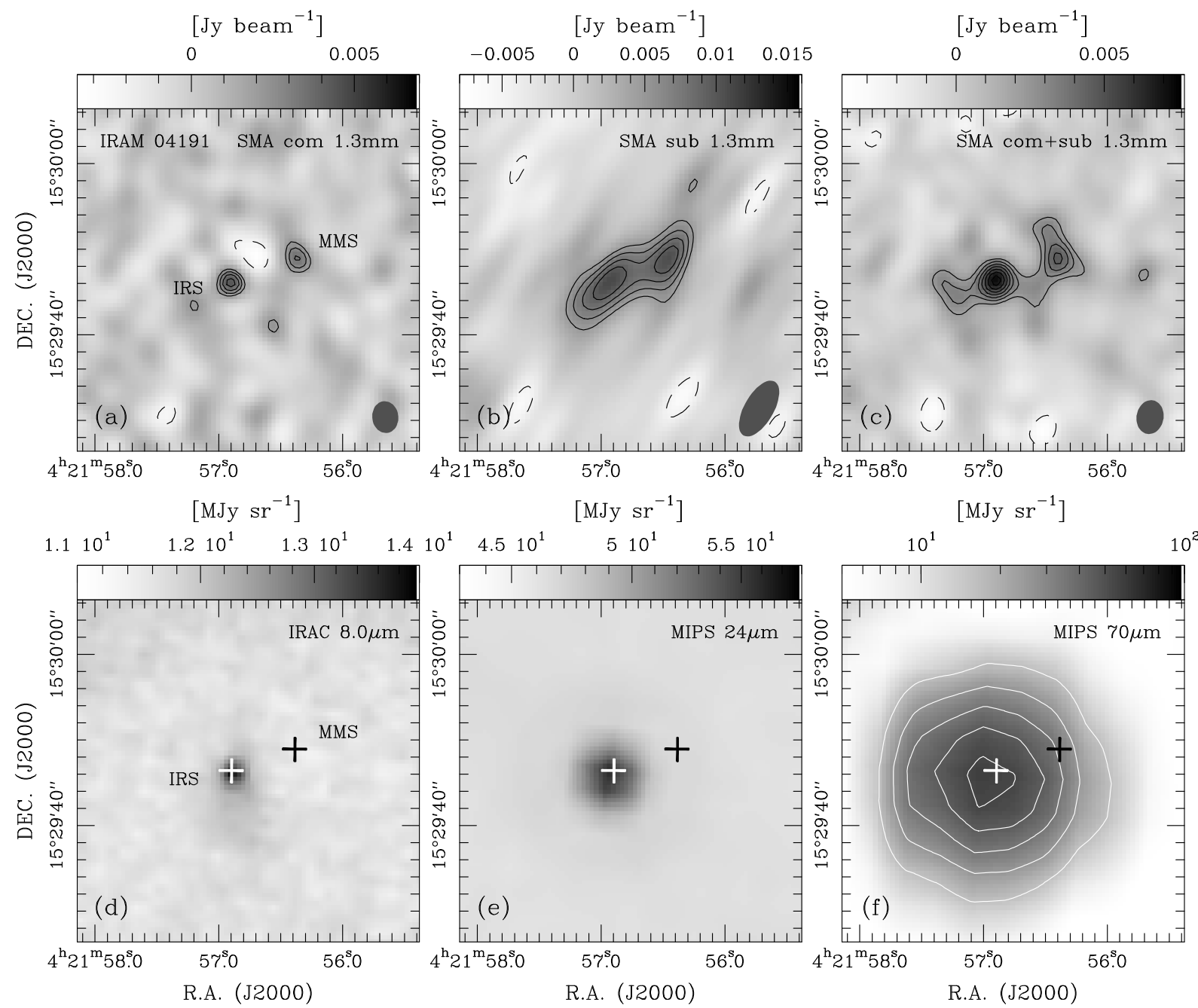

Fig. 1.- (a) SMA $1.3 \mathrm{~mm}$ dust continuum image of IRAM04191, taken with the SMA compact configuration. The contours start at $\pm 3 \sigma$, and then increase from $+3 \sigma$ in steps of $\sim 1 \sigma\left(1 \sigma \sim 0.70 \mathrm{mJy}\right.$ beam $\left.^{-1}\right)$. The synthesized SMA beam is shown as a grey oval in the bottom right corner. (b) Same as Figure a, but taken with the SMA subcompact configuration $\left(1 \sigma \sim 1.6 \mathrm{mJy}_{\text {beam }}{ }^{-1}\right)$. (c) The SMA $1.3 \mathrm{~mm}$ continuum image, using the combined compact and subcompact data $\left(1 \sigma \sim 0.77 \mathrm{mJy}_{\text {beam }^{-1}}\right)$. (d) The Spitzer IRAC $8.0 \mu \mathrm{m} \mathrm{im-}$ age of IRAM 04191. The two crosses show the positions of the two SMA continuum sources IRS (white) and MMS (black). (e) Same as Figure d, but the MIPS $24 \mu \mathrm{m}$ image. (f) Same as Figure d, but the MIPS $70 \mu \mathrm{m}$ image (the contours start at $16 \mathrm{MJy} \mathrm{sr}^{-1}$, and increase in steps of $10 \mathrm{MJy} \mathrm{sr}^{-1}$ ). The Spitzer data of IRAM 04191 are adopted from Dunham et al. (2006). 


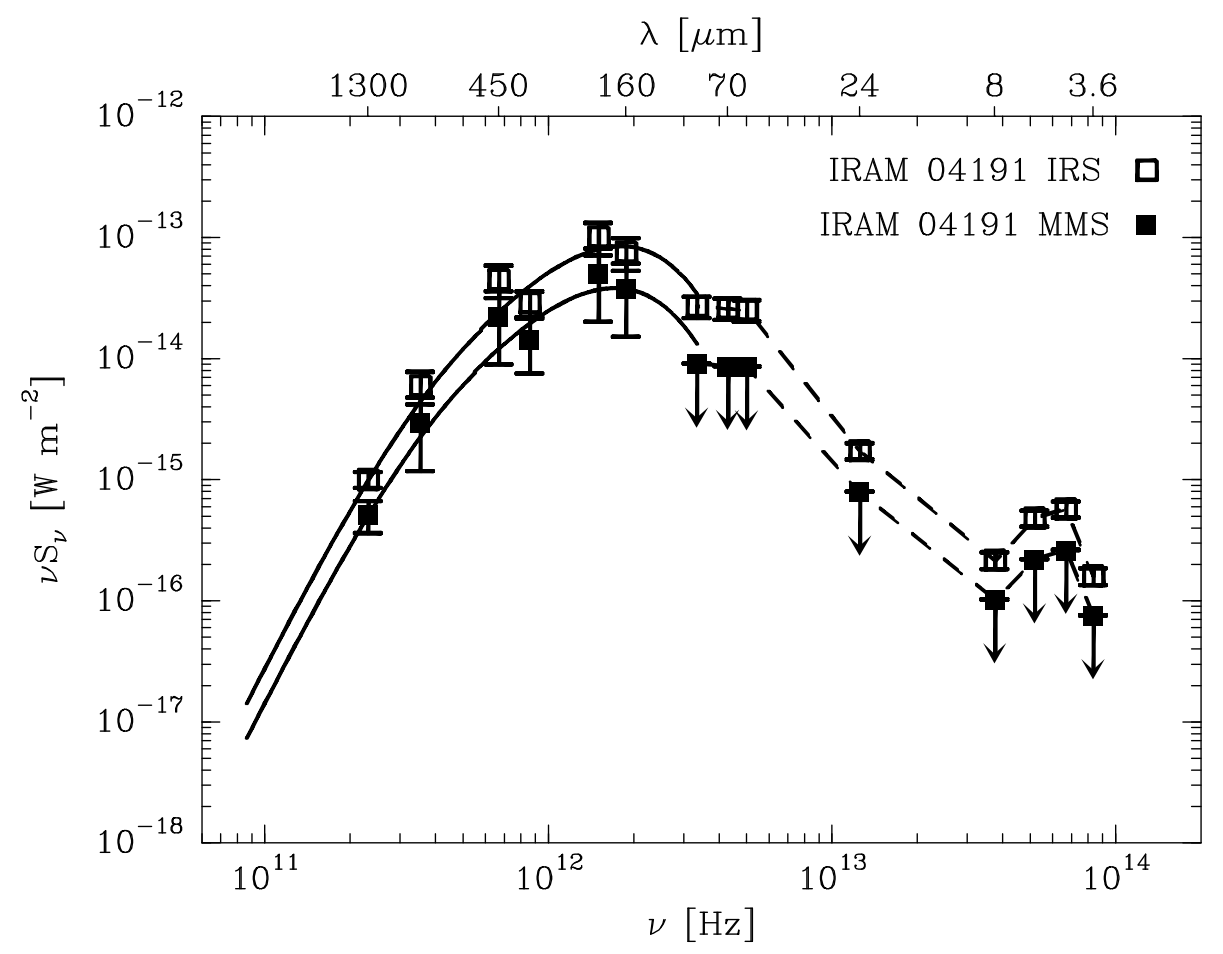

Fig. 2.- Spectral energy distributions of IRAM 04191 IRS and MMS. Solid lines show the best-fit for all points at $\lambda>90 \mu \mathrm{m}$ using a grey-body model $S_{\nu}=B_{\nu}\left(T_{\mathrm{d}}\right)\left(1-e^{-\tau_{\nu}}\right) \Omega$, where $B_{\nu}\left(T_{\mathrm{d}}\right)$ is the Planck function at frequency $\nu$ and dust temperature $T_{\mathrm{d}}, \tau_{\nu}$ is the dust optical depth as a function of frequency $\tau \propto \nu^{1.8}$, and $\Omega$ is the solid angle of the source. Dashed lines show a simple logarithmic interpolation performed between all points at $\lambda \leq 90 \mu \mathrm{m}$ for the two sources (the datapoints of MMS at wavelengths $\leq 90 \mu \mathrm{m}$ are $3 \sigma$ upper limits derived from the infrared images). 


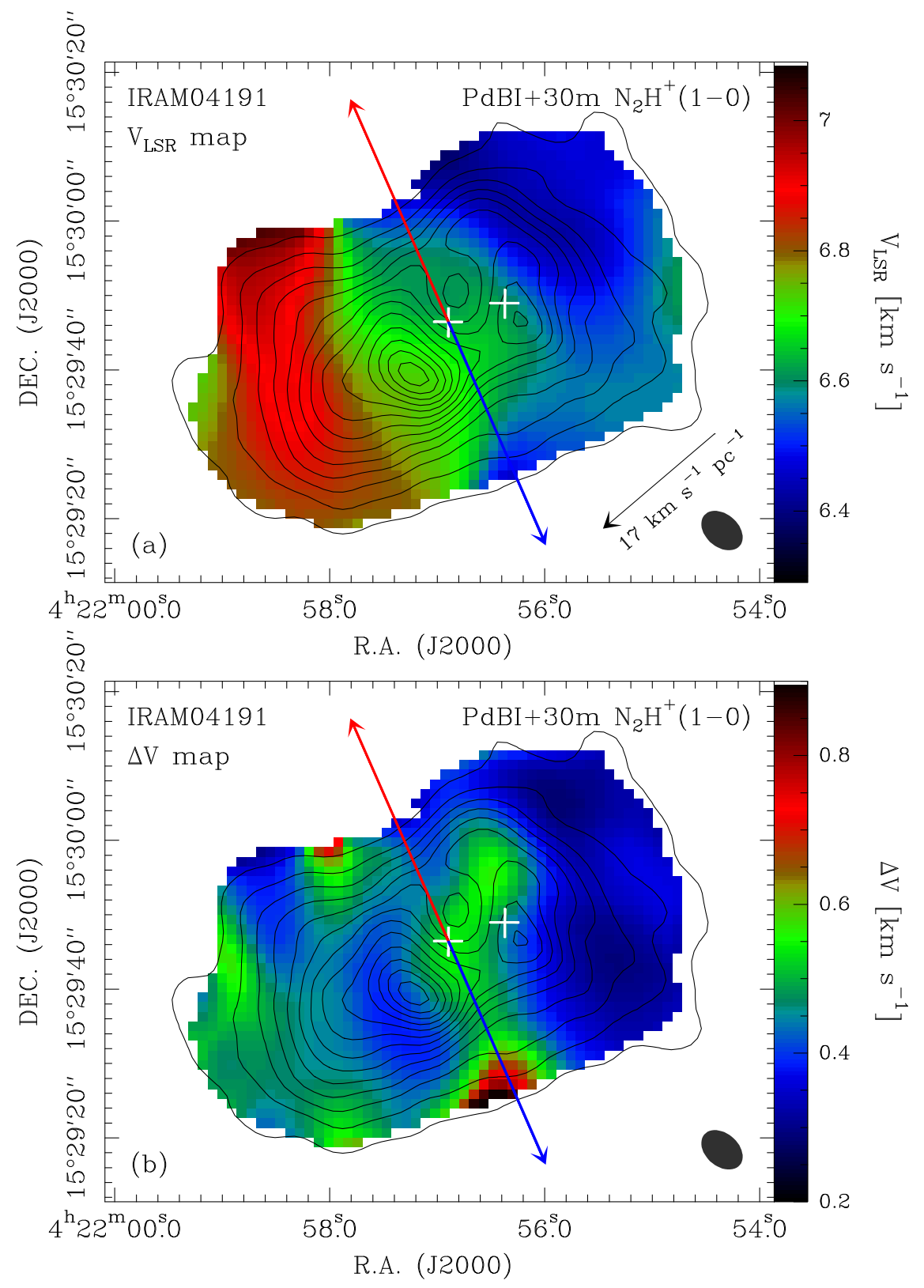

Fig. 3.- $\mathrm{N}_{2} \mathrm{H}^{+}(1-0)$ velocity fields of IRAM 04191+1522, derived from the $\mathrm{N}_{2} \mathrm{H}^{+}$data combining the IRAM-30m and PdBI observations (data from Belloche \& André 2004). The top map shows the mean velocity field (color shades), while the bottom map shows the spatial distribution of line widths (color shades). In both maps, black contours show the integrated intensity, from 0.16 to 2.1 in steps of $0.16 \mathrm{Jy}_{\text {beam }}^{-1} \mathrm{~km} \mathrm{~s}^{-1}$ ( $\sim 6$ times the rms noise); white crosses show the positions of the SMA continuum sources IRS and MMS; red and blue arrows show the directions of the red- and blue-shifted CO outflow lobes. The synthesized cleaned beam in the $\mathrm{N}_{2} \mathrm{H}^{+}$observations is shown in the bottom right corner. In the mean velocity map, the black arrow in the bottom right shows the direction of the velocity gradient $\left(\right.$ P.A. $\sim 133^{\circ}$ ). 


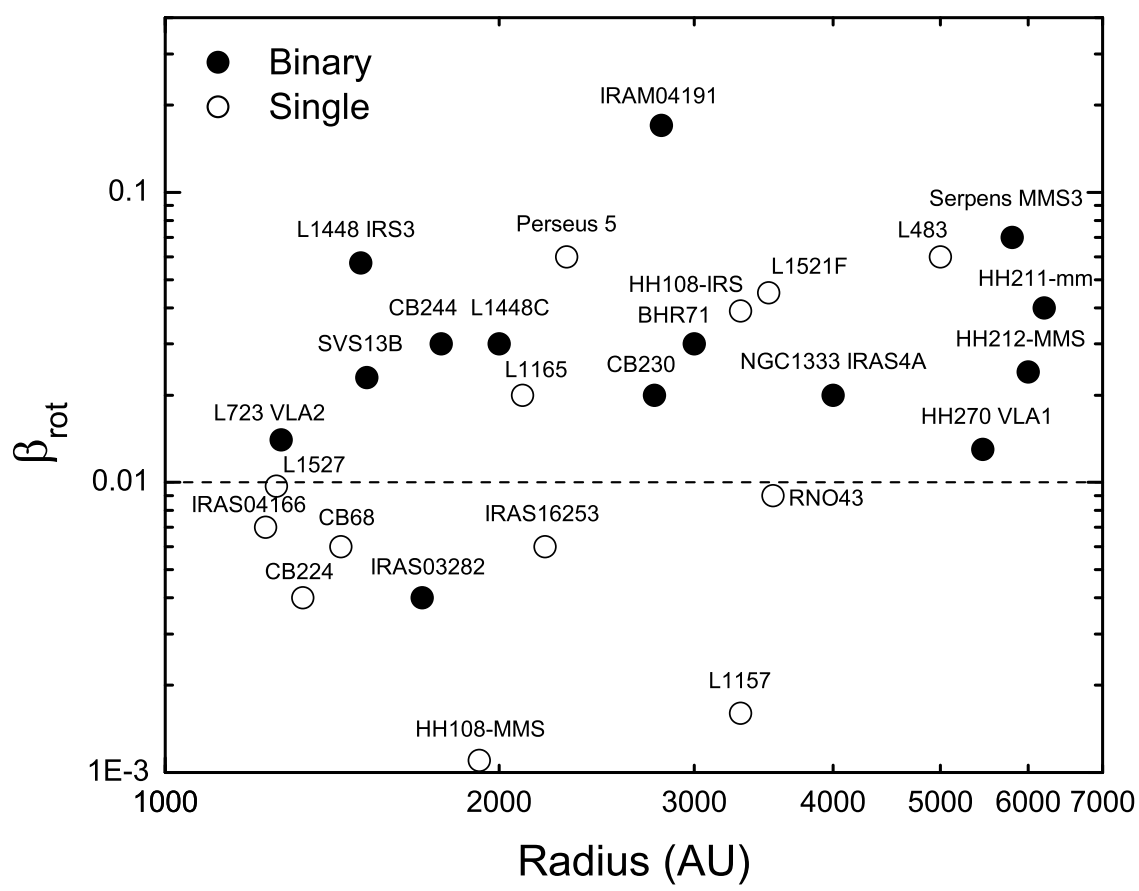

Fig. 4. - Ratio of rotational energy to gravitational energy $\beta_{\text {rot }}$ vs. core FWHM radius. Data for the sources shown are adopted from Chen et al. (2007; 2008; 2009) and Tobin et al. (2011), except IRAM 04191 (this work), NGC 1333 IRAS 4A (Belloche et al. 2006), HH 212MMS (Wiseman et al. 2001), HH 211-mms (Tanner \& Arce 2011), and L1448C/L1448 IRS3 (X. Chen et al. in preparation). Note that except for IRAM 04191, the values of $\beta_{\text {rot }}$ in this plot are lower limits, as they are not corrected for inclination. 